Radiative decay rates for W I, W II and W III allowed and forbidden transitions of interest for spectroscopic diagnostics in fusion plasmas

This article has been downloaded from IOPscience. Please scroll down to see the full text article.

2010 J. Phys. B: At. Mol. Opt. Phys. 43144003

(http://iopscience.iop.org/0953-4075/43/14/144003)

View the table of contents for this issue, or go to the journal homepage for more

Download details:

IP Address: 81.242.143.128

The article was downloaded on 17/07/2010 at 08:48

Please note that terms and conditions apply. 


\title{
Radiative decay rates for W I, W II and W III allowed and forbidden transitions of interest for spectroscopic diagnostics in fusion plasmas
}

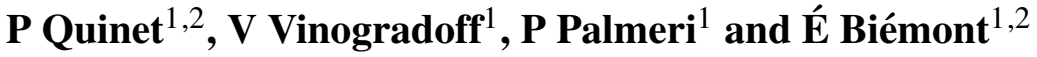 \\ ${ }^{1}$ Astrophysique et Spectroscopie, Université de Mons-UMONS, B-7000 Mons, Belgium \\ 2 IPNAS, Université de Liège, B15 Sart Tilman, B-4000 Liège, Belgium \\ E-mail: quinet@umons.ac.be
}

Received 12 November 2009, in final form 20 January 2010

Published 5 July 2010

Online at stacks.iop.org/JPhysB/43/144003

\begin{abstract}
Transition probabilities for allowed and forbidden lines in neutral, singly ionized and doubly ionized tungsten are discussed in the present paper. For the electric dipole transitions, recommended values are proposed from a critical evaluation of the data available in the literature. For the magnetic dipole and electric quadrupole transitions, for which no data have been published so far, a new set of radiative rates has been obtained using a relativistic Hartree-Fock approach including core-polarization effects. The tables summarizing the compiled data are expected to be useful for plasma modelling in fusion reactors.
\end{abstract}

\section{Introduction}

Because of its high melting point $\left(3410^{\circ} \mathrm{C}\right)$ and thermal conductivity, and its low tritium retention and erosion rate under plasma loading (see e.g. Federici et al 2001, Pospieszczyk 2006), tungsten is a very attractive element to be used as a plasma facing material in tokamak devices. The international thermonuclear experimental reactor ITER will be the next step experimental fusion device which will use tungsten, together with beryllium and carbon-fiber reinforced composite, as plasma facing materials. The main disadvantage of tungsten in these conditions is the large radiative loss due to plasma contamination and its high radiative efficiency.

Several experiments were also carried out at Alcator Cmod (Lipschultz et al 2001) and at ASDEX-U (Neu et al 2005) to prove the suitability of $\mathrm{W}$ as a divertor target material under the conditions of a high-density and low-temperature divertor.

In tokamak devices, tungsten will be sputtered from the plasma wall as a neutral element and the intensity of the well-known W I emission line at $400.8753 \mathrm{~nm}$ may be used to estimate tungsten influx (Skinner 2008). Unfortunately, as already mentioned by the latter author, a complication arises from the fact that there exists a coincident W II line at $400.8751 \mathrm{~nm}$. Another difficulty originates from the fact that sputtered high- $Z$ metal atoms, such as tungsten, have a low ionization potential $(7.864 \mathrm{eV})$ and a relatively low velocity. They are quickly ionized close to the surface and most ions are promptly redeposited onto the wall near their point of origin (Skinner 2009).

Estimates of the tungsten influx rate to the core plasma will depend on a calculation of transport from the wall surface through the scrape-off layer. Consequently, the identification of emission lines from neutral and lowly ionized tungsten will greatly aid modelling of the plasma edge and scrape-off layer transport and facilitate the analysis of net tungsten influx rates.

However, a very small number of contributions have been devoted, in the past, to the determination of radiative data for the lower charge states of tungsten. The aim of the present paper is to report on the best transition rates for selected electric dipole (E1) transitions and to fill in the gap regarding the transition probabilities for forbidden (E2 and M1) lines in neutral (W I), singly ionized (W II) and doubly ionized (W III) tungsten. 


\section{Allowed transitions}

\subsection{W I lines}

Wavelengths of all the transitions observed in the W I spectrum, and energy levels derived from these wavelengths, were compiled by Kramida and Shirai (2006) who critically evaluated the data published previously by Laun and Corliss (1968), Shadmi and Caspi (1968), Corliss (1969), Wyart (1978), Martin et al (1978) and Campbell-Miller and Simard (1996).

Experimental transition probabilities in W I were first determined by Corliss and Bozman (1962) but their arc measurements later on were recognized to be affected by large systematic errors. These results were supplemented by the relative measurements of Clawson and Miller (1973) and by the absolute measurements of Obbarius and Kock (1982). In the latter case, a stabilized arc, operated in argon, was used for measuring oscillator strengths for $43 \mathrm{~W}$ I lines in the wavelength range $240-560 \mathrm{~nm}$.

The first radiative lifetime measurements in $\mathrm{W}$ I were performed for 15 levels belonging to the $5 \mathrm{~d}^{4} 6 \mathrm{~s} 6 \mathrm{p}$ and $5 d^{5} 6 p$ configurations by Duquette et al (1981) who used time-resolved laser-induced fluorescence (TR-LIF) and a hollow cathode effusive atomic beam source. Kwiatkowski et al (1982) reported lifetimes for 13 energy levels in the configurations $(5 d+6 s)^{5} 6 \mathrm{p}$. These measurements were based on the observation of the re-emitted fluorescence with a singlephoton-counting technique after a selective excitation of an atomic beam by a pulsed dye laser. Some radiative lifetimes of W I excited states were also published by Plekhotkin and Verolainen (1985).

The lifetimes by Schnabel and Kock (1997), obtained with the TR-LIF method for $47 \mathrm{~W}$ I levels in the energy range $27800-48200 \mathrm{~cm}^{-1}$, agree within the mutual uncertainties with the results of Den Hartog et al (1987) (three lifetimes common to both works). The lifetimes by Kwiatkowski et al agree within 7\% with those of Den Hartog et al (1987) (13 levels in common), the latter work including remeasurements of the values published by Duquette et al (1981). It is thus obvious that the three scales of published lifetimes measured with reliable techniques (laser spectroscopy) are in excellent agreement.

The most recent and extensive sets of experimental decay rates in neutral tungsten were reported by Den Hartog et al (1987) and Kling and Kock (1999) who measured branching fractions (BF) on high-resolution Fourier transform spectra and were able to deduce absolute transition probabilities for a set of 572 lines covering the wavelength range $225-1035 \mathrm{~nm}$ and involving excited energy levels up to $46932 \mathrm{~cm}^{-1}$. The lifetimes used in these two papers were those of Den Hartog et al (1987) and of Schnabel and Kock (1997), respectively. As the work of Kling and Kock (1999) was focused on higher lying levels than that of Den Hartog et al (1987), the overlap of the two works is small. In fact, BFs were measured for only 19 lines in common. The agreement is within a few $(<7) \%$ for the most intense transitions ( $\mathrm{BF}>20 \%$ ), larger discrepancies appearing (as expected) for some weaker lines. In the present work, we have adopted the transition probabilities reported by Den Hartog et al (1987) and Kling and Kock (1999). According to these authors, the uncertainties affecting their results are smaller than $10 \%$ for the majority of the transitions.

We report in table 1 a list of selected lines of W I covering the UV and visible regions and suitable for plasma diagnostics. This line list has been established by adopting the following criteria. The lines are unblended according to the wavelength compilation of Kramida and Shirai (2006). The list is also limited to the strongest transitions, i.e. the transitions such that the laboratory intensities (Int.) $\geqslant 100$. We are conscious however that this choice has its own limitations and is partly dependent upon the physical conditions met in the light source used for establishing the intensity scale. For all the selected transitions, reliable transition probabilities and oscillator strengths are available. They are due to Den Hartog et al (1987) and Kling and Kock (1999). It is seen, from the last column of table 1 , that these two sets of transition probabilities agree quite well for the six transitions common to both works.

It should be emphasized that the well-known W I emission line at $400.8753 \mathrm{~nm}$, tentatively used for plasma diagnostics as stated in the introduction, has been kept in table 1 despite of the fact that it is blended with a W II line appearing at $400.8751 \mathrm{~nm}$. This line is among the strongest observed in the laboratory and, for that reason, deserves to be considered with a special attention (see the discussion in section 2.2).

\subsection{W II lines}

76 even levels and 187 odd levels belonging to singly ionized tungsten were reported in the compilation of Kramida and Shirai (2006) which was essentially based on the extensive investigations of the W II spectrum by Ekberg et al (2000) and by Cabeza et al (1985).

After the pioneering work by Corliss and Bozman (1962), relative and absolute transition probabilities were determined by Clawson and Miller (1973) and Obbarius and Kock (1982), respectively. The latter authors published oscillator strengths for 27 W II transitions between 240 and $560 \mathrm{~nm}$.

Substantial progress in radiative parameter determination in W II resulted from the combination of lifetime measurements with $\mathrm{BF}$ determinations. Different sets of lifetimes were reported. Kwiatkowski et al (1984) measured three lifetimes using the TR-LIF method, the ions being produced by the sputtering technique in a low-pressure discharge. Using the TR-LIF technique, radiative lifetime measurements were also carried out by Schnabel et al (1998) for 19 selected levels with energies between 36000 and $55000 \mathrm{~cm}^{-1}$ and by Schulz-Johanning et al (1999) who used a linear Paul trap for investigating two W II lifetimes. More recently, Henderson et al (1999) reported three lifetime values using the beam-foil method. These results were combined with theoretical and experimental BFs deduced from Corliss and Bozman (1962) arc measurements.

The lifetimes by Schnabel et al (1998) agree, within the quoted uncertainties, with the results of Kwiatkowski et al (1984) for two levels, the measurement reported by these authors for $42390.27 \mathrm{~cm}^{-1}$ being obviously in error 
Table 1. UV and visible transitions of neutral tungsten (W I) suitable for plasma diagnostics. For the choice of the lines, see the text.

\begin{tabular}{|c|c|c|c|c|c|c|c|}
\hline $\begin{array}{l}\lambda^{\mathrm{a}} \\
(\mathrm{nm})\end{array}$ & $\begin{array}{l}E_{\text {low }}^{\mathrm{b}} \\
\left(\mathrm{cm}^{-1}\right)\end{array}$ & $\mathrm{J}$ & $\begin{array}{l}E_{\text {upp. }}^{\mathrm{b}} \\
\left(\mathrm{cm}^{-1}\right)\end{array}$ & $J^{\prime}$ & Int. $^{b}$ & $\log g f$ & $\begin{array}{l}g A \\
\left(s^{-1}\right)\end{array}$ \\
\hline 245.1996 & 0.00 & 0 & 40770.78 & 1 & 100 & $-0.68^{c}$ & $2.34(8)^{\mathrm{c}}$ \\
\hline 245.6534 & 3325.53 & 2 & 44021.00 & 3 & 150 & $-0.53^{c}$ & $3.30(8)^{\mathrm{c}}$ \\
\hline 245.9300 & 3325.53 & 2 & 43975.22 & 2 & 140 & $-0.41^{\mathrm{c}}$ & $4.31(8)^{\mathrm{c}}$ \\
\hline 246.6848 & 3325.53 & 2 & 43850.84 & 3 & 100 & $-0.35^{\mathrm{c}}$ & $4.52(8)^{c}$ \\
\hline 247.4149 & 6219.33 & 4 & 46625.05 & 4 & 140 & $-0.01^{\mathrm{c}}$ & $1.07(9)^{\mathrm{c}}$ \\
\hline 248.0130 & 1670.29 & 1 & 41978.62 & 2 & 100 & $-0.67^{\mathrm{c}}$ & $2.35(8)^{\mathrm{c}}$ \\
\hline 248.1440 & 6219.33 & 4 & 46506.37 & 5 & 150 & $0.18^{\mathrm{c}}$ & $1.63(9)^{\mathrm{c}}$ \\
\hline 249.5264 & 1670.29 & 1 & 41734.13 & 2 & 100 & $-0.66^{\mathrm{c}}$ & $2.33(8)^{\mathrm{c}}$ \\
\hline 254.7136 & 3325.53 & 2 & 42573.49 & 1 & 100 & $-0.29^{c}$ & $5.25(8)^{c}$ \\
\hline 255.1349 & 0.00 & 0 & 39183.20 & 1 & 200 & $-0.28^{c}$ & $5.34(8)^{\mathrm{c}}$ \\
\hline 255.3824 & 4830.00 & 3 & 43975.22 & 2 & 100 & $-0.68^{c}$ & $2.14(8)^{\mathrm{c}}$ \\
\hline 256.1968 & 4830.00 & 3 & 43850.84 & 3 & 100 & $-0.48^{c}$ & $3.35(8)^{\mathrm{c}}$ \\
\hline 258.0487 & 1670.29 & 1 & 40411.12 & 1 & 125 & $-0.65^{c}$ & $2.25(8)^{\mathrm{c}}$ \\
\hline 263.3129 & 1670.29 & 1 & 39636.62 & 1 & 150 & $-0.69^{c}$ & $1.96(8)^{\mathrm{c}}$ \\
\hline 265.6540 & 2951.29 & 3 & 40583.07 & 4 & 300 & $-0.19^{c}$ & $6.07(8)^{\mathrm{c}}$ \\
\hline 266.2835 & 3325.53 & 2 & 40868.40 & 2 & 200 & $-0.65^{c}$ & $2.11(8)^{\mathrm{c}}$ \\
\hline 266.4966 & 1670.29 & 1 & 39183.20 & 1 & 100 & $-1.25^{\mathrm{c}}$ & $5.34(7)^{\mathrm{c}}$ \\
\hline 267.1472 & 4830.00 & 3 & 42251.51 & 3 & 200 & $-0.53^{c}$ & $2.74(8)^{\mathrm{c}}$ \\
\hline 267.7276 & 3325.53 & 2 & 40665.85 & 3 & 100 & $-0.92^{c}$ & $1.11(8)^{\mathrm{c}}$ \\
\hline 268.1422 & 2951.29 & 3 & 40233.97 & 4 & 400 & $-0.15^{\mathrm{c}}$ & $6.62(8)^{c}$ \\
\hline 269.1094 & 4830.00 & 3 & 41978.62 & 2 & 100 & $-1.14^{\mathrm{c}}$ & $6.65(7)^{\mathrm{c}}$ \\
\hline 269.567 & 3325.53 & 2 & 40411.12 & 1 & 150 & $-0.81^{\mathrm{c}}$ & $1.41(8)^{\mathrm{c}}$ \\
\hline 269.9594 & 6219.33 & 4 & 43251.00 & 4 & 150 & $-0.42^{\mathrm{c}}$ & $3.42(8)^{\mathrm{c}}$ \\
\hline 271.8906 & 2951.29 & 3 & 39719.96 & 4 & 250 & $-0.33^{\mathrm{c}}$ & $4.26(8)^{c}$ \\
\hline 272.4352 & 2951.29 & 3 & 39646.41 & 3 & 300 & $-0.09^{c}$ & $7.35(8)^{\mathrm{c}}$ \\
\hline 276.2339 & 0.00 & 0 & 36190.49 & 1 & 150 & $-1.20^{\mathrm{c}}$ & $5.52(7)^{\mathrm{c}}$ \\
\hline 277.0880 & 2951.29 & 3 & 39030.25 & 2 & 200 & $-0.98^{c}$ & $9.15(7)^{\mathrm{c}}$ \\
\hline 277.3999 & 4830.00 & 3 & 40868.40 & 2 & 200 & $-0.64^{\mathrm{c}}$ & $1.97(8)^{\mathrm{c}}$ \\
\hline 277.4476 & 6219.33 & 4 & 42251.51 & 3 & 300 & $-0.42^{\mathrm{c}}$ & $3.33(8)^{\mathrm{c}}$ \\
\hline 281.8060 & 6219.33 & 4 & 41694.34 & 3 & 250 & $-0.44^{\mathrm{c}}$ & $3.02(8)^{\mathrm{c}}$ \\
\hline 283.1379 & 2951.29 & 3 & 38259.40 & 4 & 250 & $-0.27^{\mathrm{c}}$ & $4.42(8)^{\mathrm{c}}$ \\
\hline 283.3630 & 6219.33 & 4 & 41499.43 & 3 & 120 & $-0.40^{\mathrm{c}}$ & $3.35(8)^{\mathrm{c}}$ \\
\hline 287.9396 & 0.00 & 0 & 34719.33 & 1 & 140 & $-1.04^{\mathrm{c}}$ & $7.38(7)^{\mathrm{c}}, 7.3(7)^{\mathrm{d}}$ \\
\hline 289.6009 & 1670.29 & 1 & 36190.49 & 1 & 150 & $-0.98^{c}$ & $8.40(7)^{\mathrm{c}}$ \\
\hline 291.0997 & 0.00 & 0 & 34342.44 & 1 & 100 & $-1.56^{\mathrm{c}}$ & $2.17(7)^{\mathrm{c}}, 2.3(7)^{\mathrm{d}}$ \\
\hline 292.3103 & 4830.00 & 3 & 39030.25 & 2 & 150 & $-1.31^{\mathrm{c}}$ & $3.83(7)^{\mathrm{c}}$ \\
\hline 293.4996 & 1670.29 & 1 & 35731.96 & 2 & 250 & $-1.03^{\mathrm{d}}$ & $7.3(7)^{\mathrm{d}}$ \\
\hline 294.4398 & 2951.29 & 3 & 36904.16 & 2 & 300 & $-0.15^{\mathrm{c}}$ & $5.40(8)^{\mathrm{c}}$ \\
\hline 294.6989 & 2951.29 & 3 & 36874.36 & 3 & 300 & $-0.12^{\mathrm{c}}$ & $5.76(8)^{\mathrm{c}}$ \\
\hline 301.3788 & 4830.00 & 3 & 38001.12 & 4 & 120 & $-1.10^{\mathrm{d}}$ & $5.8(7)^{\mathrm{d}}$ \\
\hline 301.6466 & 6219.33 & 4 & 39361.01 & 5 & 150 & $-0.86^{\mathrm{d}}$ & $1.02(8)^{\mathrm{d}}$ \\
\hline 301.7436 & 2951.29 & 3 & 36082.30 & 4 & 200 & $-0.83^{\mathrm{d}}$ & $1.09(8)^{\mathrm{d}}$ \\
\hline 302.4928 & 1670.29 & 1 & 34719.33 & 1 & 100 & $-1.19^{c}$ & $4.74(7)^{\mathrm{c}}, 4.2(7)^{\mathrm{d}}$ \\
\hline 304.6440 & 1670.29 & 1 & 34485.86 & 2 & 120 & $-1.39^{\mathrm{d}}$ & $2.9(7)^{\mathrm{d}}$ \\
\hline 304.9688 & 2951.29 & 3 & 35731.96 & 2 & 120 & $-0.92^{\mathrm{d}}$ & $8.6(7)^{\mathrm{d}}$ \\
\hline 309.3500 & 4830.00 & 3 & 37146.36 & 4 & 100 & $-1.24^{\mathrm{d}}$ & $4.0(7)^{\mathrm{d}}$ \\
\hline 310.7227 & 3325.53 & 2 & 35499.15 & 3 & 100 & $-1.63^{\mathrm{d}}$ & $1.63(7)^{\mathrm{d}}$ \\
\hline 310.8018 & 2951.29 & 3 & 35116.78 & 4 & 100 & $-1.69^{\mathrm{d}}$ & $1.42(7)^{\mathrm{d}}$ \\
\hline 317.6601 & 1670.29 & 1 & 33141.38 & 2 & 150 & $-1.79^{d}$ & $1.06(7)^{\mathrm{d}}$ \\
\hline 318.4418 & 3325.53 & 2 & 34719.33 & 1 & 120 & $-1.94^{\mathrm{c}}$ & $7.59(6)^{\mathrm{c}}, 7.0(6)^{\mathrm{d}}$ \\
\hline 319.1572 & 0.00 & 0 & 31323.48 & 1 & 200 & $-1.83^{\mathrm{d}}$ & $9.7(6)^{\mathrm{d}}$ \\
\hline 319.8840 & 4830.00 & 3 & 36082.30 & 4 & 200 & $-1.20^{\mathrm{d}}$ & $4.1(7)^{\mathrm{d}}$ \\
\hline 320.725 & 2951.29 & 3 & 34121.68 & 4 & 300 & $-1.38^{\mathrm{d}}$ & $2.7(7)^{\mathrm{d}}$ \\
\hline 320.828 & 3325.53 & 2 & 34485.86 & 2 & 200 & $-1.47^{\mathrm{d}}$ & $2.2(7)^{\mathrm{d}}$ \\
\hline 321.5562 & 6219.33 & 4 & 37309.16 & 5 & 200 & $-0.45^{\mathrm{d}}$ & $2.3(8)^{\mathrm{d}}$ \\
\hline 330.0822 & 4830.00 & 3 & 35116.78 & 4 & 150 & $-0.92^{\mathrm{d}}$ & $7.3(7)^{\mathrm{d}}$ \\
\hline 331.1388 & 2951.29 & 3 & 33141.38 & 2 & 200 & $-1.34^{\mathrm{d}}$ & $2.8(7)^{\mathrm{d}}$ \\
\hline 334.5858 & 13348.56 & 3 & 43227.66 & 2 & 100 & $-1.11^{\mathrm{c}}$ & $4.60(7)^{c}$ \\
\hline 353.5539 & 13307.10 & 1 & 41583.20 & 2 & 100 & $-1.15^{\mathrm{c}}$ & $3.78(7)^{\mathrm{c}}$ \\
\hline 354.5220 & 0.00 & 0 & 28198.90 & 1 & 200 & $-1.74^{\mathrm{d}}$ & $9.6(6)^{d}$ \\
\hline 360.6063 & 1670.29 & 1 & 29393.40 & 2 & 150 & $-2.03^{\mathrm{d}}$ & $4.8(6)^{\mathrm{d}}$ \\
\hline 361.7515 & 2951.29 & 3 & 30586.64 & 3 & 500 & $-0.83^{\mathrm{d}}$ & $7.5(7)^{\mathrm{d}}$ \\
\hline 363.1943 & 1670.29 & 1 & 29195.84 & 2 & 200 & $-1.88^{\mathrm{d}}$ & $6.6(6)^{\mathrm{d}}$ \\
\hline 368.2084 & 6219.33 & 4 & 33370.04 & 5 & 200 & $-1.35^{\mathrm{d}}$ & $2.2(7)^{\mathrm{d}}$ \\
\hline
\end{tabular}


Table 1. (Continued.)

\begin{tabular}{|c|c|c|c|c|c|c|c|}
\hline $\begin{array}{l}\lambda^{\mathrm{a}} \\
(\mathrm{nm})\end{array}$ & $\begin{array}{l}E_{\mathrm{low}}^{\mathrm{b}} \\
\left(\mathrm{cm}^{-1}\right)\end{array}$ & $\mathrm{J}$ & $\begin{array}{l}E_{\text {upp. }}^{\mathrm{b}} \\
\left(\mathrm{cm}^{-1}\right)\end{array}$ & $J^{\prime}$ & Int. $^{b}$ & $\log g f$ & $\begin{array}{l}g A \\
\left(\mathrm{~s}^{-1}\right)\end{array}$ \\
\hline 370.7922 & 2951.29 & 3 & 29912.85 & 3 & 300 & $-1.38^{\mathrm{d}}$ & $2.0(7)^{\mathrm{d}}$ \\
\hline 376.0126 & 3325.53 & 2 & 29912.85 & 3 & 200 & $-1.53^{\mathrm{d}}$ & $1.39(7)^{\mathrm{d}}$ \\
\hline 376.8447 & 1670.29 & 1 & 28198.90 & 1 & 250 & $-1.65^{\mathrm{d}}$ & $1.04(7)^{\mathrm{d}}$ \\
\hline 378.0772 & 2951.29 & 3 & 29393.40 & 2 & 200 & $-1.35^{\mathrm{d}}$ & $2.1(7)^{\mathrm{d}}$ \\
\hline 380.9220 & 2951.29 & 3 & 29195.84 & 2 & 150 & $-2.01^{\mathrm{d}}$ & $4.5(6)^{\mathrm{d}}$ \\
\hline 381.0790 & 13777.71 & 2 & 40011.50 & 2 & 120 & $-1.14^{\mathrm{c}}$ & $3.30(7)^{\mathrm{c}}$ \\
\hline 381.7480 & 2951.29 & 3 & 29139.12 & 3 & 400 & $-1.32^{\mathrm{d}}$ & $2.2(7)^{\mathrm{d}}$ \\
\hline 383.5058 & 3325.53 & 2 & 29393.40 & 2 & 250 & $-1.24^{\mathrm{d}}$ & $2.6(7)^{\mathrm{d}}$ \\
\hline 384.6218 & 1670.29 & 1 & 27662.52 & 2 & 300 & $-1.62^{\mathrm{d}}$ & $1.07(7)^{\mathrm{d}}$ \\
\hline 384.7498 & 0.00 & 0 & 25983.60 & 1 & 150 & $-2.26^{\mathrm{d}}$ & $2.5(6)^{d}$ \\
\hline 386.7986 & 2951.29 & 3 & 28797.24 & 4 & 600 & $-1.04^{\mathrm{d}}$ & $4.1(7)^{d}$ \\
\hline 388.1405 & 4830.00 & 3 & 30586.64 & 3 & 250 & $-1.25^{\mathrm{d}}$ & $2.5(7)^{\mathrm{d}}$ \\
\hline $400.8753^{\star}$ & 2951.29 & 3 & 27889.68 & 4 & 1000 & $-0.44^{\mathrm{c}}$ & $1.50(8)^{c}, 1.47(8)^{d}$ \\
\hline 404.5594 & 2951.29 & 3 & 27662.52 & 2 & 100 & $-1.45^{\mathrm{d}}$ & $1.44(7)^{\mathrm{d}}$ \\
\hline 407.4358 & 2951.29 & 3 & 27488.11 & 3 & 600 & $-0.74^{\mathrm{d}}$ & $7.3(7)^{\mathrm{d}}$ \\
\hline 410.2702 & 6219.33 & 4 & 30586.64 & 3 & 100 & $-1.07^{\mathrm{d}}$ & $3.4(7)^{\mathrm{d}}$ \\
\hline 424.1445 & 15460.01 & 3 & 39030.25 & 2 & 100 & $-0.84^{\mathrm{c}}$ & $5.35(7)^{\mathrm{c}}$ \\
\hline 424.4364 & 6219.33 & 4 & 29773.34 & 5 & 200 & $-1.39^{\mathrm{d}}$ & $1.52(7)^{\mathrm{d}}$ \\
\hline 426.9382 & 2951.29 & 3 & 26367.28 & 2 & 200 & $-1.38^{\mathrm{d}}$ & $1.52(7)^{\mathrm{d}}$ \\
\hline 429.4606 & 2951.29 & 3 & 26229.77 & 2 & 800 & $-0.77^{\mathrm{c}}$ & $6.20(7)^{\mathrm{c}}, 6.2(7)^{\mathrm{d}}$ \\
\hline 430.2110 & 2951.29 & 3 & 26189.20 & 3 & 200 & $-1.16^{\mathrm{d}}$ & $2.5(7)^{\mathrm{d}}$ \\
\hline 484.3810 & 3325.53 & 2 & 23964.67 & 2 & 400 & $-1.47^{\mathrm{d}}$ & $9.6(6)^{d}$ \\
\hline 488.6899 & 6219.33 & 4 & 26676.48 & 5 & 200 & $-1.50^{\mathrm{d}}$ & $8.9(6)^{\mathrm{d}}$ \\
\hline 498.2593 & 0.00 & 0 & 20064.30 & 1 & 200 & $-2.33^{\mathrm{d}}$ & $1.25(6)^{\mathrm{d}}$ \\
\hline 505.3276 & 1670.29 & 1 & 21453.90 & 1 & 400 & $-1.66^{\mathrm{d}}$ & $5.7(6)^{\mathrm{d}}$ \\
\hline 522.4657 & 4830.00 & 3 & 23964.67 & 2 & 250 & $-1.60^{\mathrm{d}}$ & $6.2(6)^{\mathrm{d}}$ \\
\hline 524.298 & 16431.31 & 4 & 35499.15 & 3 & 250 & $-1.50^{\mathrm{d}}$ & $7.7(6)^{\mathrm{d}}$ \\
\hline 525.4542 & 15460.01 & 3 & 34485.86 & 2 & 100 & $-2.10^{\mathrm{d}}$ & $1.93(6)^{\mathrm{d}}$ \\
\hline 525.5396 & 22476.68 & 4 & 41499.43 & 3 & 120 & $-1.12^{\mathrm{c}}$ & $1.83(7)^{\mathrm{c}}$ \\
\hline 543.5032 & 1670.29 & 1 & 20064.30 & 1 & 100 & $-2.92^{\mathrm{d}}$ & $2.7(5)^{\mathrm{d}}$ \\
\hline 551.4676 & 3325.53 & 2 & 21453.90 & 1 & 300 & $-2.00^{\mathrm{d}}$ & $2.20(6)^{\mathrm{d}}$ \\
\hline 766.488 & 18280.48 & 2 & 31323.48 & 1 & 200 & $-2.00^{\mathrm{d}}$ & $1.14(6)^{\mathrm{d}}$ \\
\hline 794.092 & 13777.71 & 2 & 26367.28 & 2 & 120 & $-2.63^{\mathrm{d}}$ & $2.5(5)^{\mathrm{d}}$ \\
\hline 801.719 & 18116.84 & 2 & 30586.64 & 3 & 120 & $-1.97^{\mathrm{d}}$ & $1.1(6)^{\mathrm{d}}$ \\
\hline 812.382 & 18280.48 & 2 & 30586.64 & 3 & 150 & $-2.16^{\mathrm{d}}$ & $7.0(5)^{\mathrm{d}}$ \\
\hline
\end{tabular}

a Observed air wavelengths taken from Kramida and Shirai (2006).

b From Kramida and Shirai (2006).

c From Kling and Kock (1999).

${ }^{\mathrm{d}}$ From Den Hartog et al (1987).

* Line blended with a W II transition (see the text).

$\mathrm{a}(\mathrm{b})$ is written for $\mathrm{a} \cdot 10^{\mathrm{b}}$.

by more than one order of magnitude. A discrepancy of $40 \%$ between the laser measurement of Schnabel et al (1998) and the beam-foil measurement of Henderson et al (1999) is observed for the level at $54229.06 \mathrm{~cm}^{-1}$, but according to the quoted uncertainties (10 and $3 \%$, respectively) and the limitations inherent to the techniques involved, the first result is expected to be the most accurate. This is confirmed, in an indirect way, by the nearly perfect agreement observed, for two levels, between the results of Schulz-Johanning et al (1999) and of Kwiatkowski et al (1984) and, in a more direct way, by the relativistic Hartree-Fock calculations including corepolarization effects (HFR+CPOL) due to Nilsson et al (2008). These authors reported in fact TR-LIF lifetime measurements for nine levels and their scale was found in excellent agreement with that of Schnabel et al (1998) when relying on the lifetime obtained for the level at $47179.94 \mathrm{~cm}^{-1}$, the only level common to both works.

BFs for $280 \mathrm{~W}$ II lines originating from 19 excited levels in the wavelength range $204-750 \mathrm{~nm}$ were obtained by Kling et al (2000) from emission measurements on a high-current hollow cathode and a Penning discharge lamp. A much more extensive set of oscillator strengths was reported by Nilsson et al (2008) who performed HFR+CPOL calculations (Quinet et al 1999) in W II. Only a sample of 290 intense transitions was published in the Nilsson et al's (2008) paper but the whole set of results (6086 transitions in the range 143-990 nm) is listed in the DESIRE database (Fivet et al 2007) on the web site http://www.umh.ac.be/ astro/desire.shtml.

As a consequence of the present discussion, it is justified to adopt as the best set of results the lifetimes by Schnabel et al (1998) and by Nilsson et al (2008) and the transition 
Table 2. UV transitions of singly ionized tungsten (W II) suitable for plasma diagnostics. For the choice of the lines, see the text.

\begin{tabular}{|c|c|c|c|c|c|c|c|}
\hline $\begin{array}{l}\lambda^{\mathrm{a}} \\
(\mathrm{nm})\end{array}$ & $\begin{array}{l}E_{\mathrm{low}}^{\mathrm{b}} \\
\left(\mathrm{cm}^{-1}\right)\end{array}$ & $\mathrm{J}$ & $\begin{array}{l}E_{\text {upp. }}^{\mathrm{b}} \\
\left(\mathrm{cm}^{-1}\right)\end{array}$ & $J^{\prime}$ & Int. $^{b}$ & $\log g f^{\mathrm{c}}$ & $\begin{array}{l}g A \\
\left(s^{-1}\right)\end{array}$ \\
\hline 178.3042 & 0.000 & $1 / 2$ & 56084.326 & $3 / 2$ & 150 & -1.54 & $6.12(7)^{c}$ \\
\hline 182.8687 & 3172.473 & $5 / 2$ & 57856.759 & $5 / 2$ & 250 & -1.08 & $1.68(8)^{\mathrm{c}}$ \\
\hline 184.8106 & 6147.085 & $9 / 2$ & 60256.547 & $7 / 2$ & 250 & -0.09 & $1.60(9)^{\mathrm{c}}$ \\
\hline 207.9118 & 6147.085 & $9 / 2$ & 54229.082 & $11 / 2$ & 296 & 0.50 & $4.86(9)^{\mathrm{c}}, 4.49(9)^{\mathrm{d}}$ \\
\hline 208.8204 & 3172.473 & $5 / 2$ & 51045.292 & $7 / 2$ & 125 & -0.02 & $1.47(9)^{\mathrm{c}}$ \\
\hline 209.4751 & 1518.829 & $3 / 2$ & 49242.042 & $5 / 2$ & 142 & -0.05 & $1.34(9)^{\mathrm{c}}, 1.06(9)^{\mathrm{d}}$ \\
\hline 215.3558 & 6147.085 & $9 / 2$ & 52567.276 & $9 / 2$ & 103 & -0.48 & $4.73(8)^{c}$ \\
\hline 216.6316 & 4716.278 & $7 / 2$ & 50863.106 & $9 / 2$ & 158 & -0.08 & $1.18(9)^{\mathrm{c}}$ \\
\hline 220.4483 & 6147.085 & $9 / 2$ & 51495.054 & $11 / 2$ & 276 & 0.17 & $2.02(9)^{\mathrm{c}}$ \\
\hline 222.5901 & 0.000 & $1 / 2$ & 44911.659 & $3 / 2$ & 162 & -0.46 & $4.66(8)^{\mathrm{c}}, 3.41(8)^{\mathrm{d}}$ \\
\hline 222.9629 & 1518.829 & $3 / 2$ & 46355.404 & $5 / 2$ & 153 & -0.41 & $5.28(8)^{\mathrm{c}}$ \\
\hline 224.8275 & 4716.278 & $7 / 2$ & 49181.034 & $9 / 2$ & 128 & -0.68 & $2.78(8)^{\mathrm{c}}$ \\
\hline 224.8758 & 0.000 & $1 / 2$ & 44455.212 & $1 / 2$ & 253 & -0.32 & $6.12(8)^{\mathrm{c}}, 6.38(8)^{\mathrm{d}}$ \\
\hline 224.9887 & 19442.466 & $13 / 2$ & 63875.361 & $15 / 2$ & 249 & 0.73 & $7.12(9)^{\mathrm{c}}$ \\
\hline 226.3519 & 17436.932 & $11 / 2$ & 61602.268 & $13 / 2$ & 127 & 0.01 & $1.33(9)^{\mathrm{c}}$ \\
\hline 227.0241 & 14857.160 & $9 / 2$ & 58891.742 & $11 / 2$ & 110 & -0.05 & $1.15(9)^{\mathrm{c}}$ \\
\hline 230.3819 & 1518.829 & $3 / 2$ & 44911.659 & $3 / 2$ & 164 & -0.56 & $3.39(8)^{\mathrm{c}}, 2.86(8)^{\mathrm{d}}$ \\
\hline 231.5018 & 3172.473 & $5 / 2$ & 46355.404 & $5 / 2$ & 122 & -0.66 & $2.70(8)^{\mathrm{c}}$ \\
\hline 232.6089 & 6147.085 & $9 / 2$ & 49124.508 & $7 / 2$ & 25 & -0.49 & \\
\hline 234.1368 & 4716.278 & $7 / 2$ & 47413.270 & $5 / 2$ & & -0.51 & \\
\hline 234.3497 & 28187.578 & $13 / 2$ & 70845.790 & $15 / 2$ & 109 & 0.15 & $1.74(9)^{\mathrm{c}}$ \\
\hline 237.0041 & 20534.191 & $11 / 2$ & 62714.675 & $13 / 2$ & 177 & 0.10 & \\
\hline 239.0370 & 7420.261 & $5 / 2$ & 49242.042 & $5 / 2$ & 110 & -0.42 & $4.38(8)^{\mathrm{c}}, 4.01(8)^{\mathrm{d}}$ \\
\hline 239.2928 & 4716.278 & $7 / 2$ & 46493.356 & $9 / 2$ & 231 & -0.62 & $2.81(8)^{\mathrm{c}}, 2.28(8)^{\mathrm{d}}$ \\
\hline 239.7079 & 3172.473 & $5 / 2$ & 44877.209 & $7 / 2$ & 294 & -0.39 & $4.76(8)^{\mathrm{c}}, 4.19(8)^{\mathrm{d}}$ \\
\hline 239.7107 & 7420.261 & $5 / 2$ & 49124.508 & $7 / 2$ & 421 & -0.59 & $2.97(8)^{\mathrm{c}}$ \\
\hline 242.7490 & 3172.472 & $5 / 2$ & 44354.784 & $5 / 2$ & & -0.76 & $1.97(8)^{\mathrm{c}}, 1.54(8)^{\mathrm{d}}$ \\
\hline 243.5003 & 20534.191 & $11 / 2$ & 61589.457 & $11 / 2$ & 10 & -0.15 & $8.02(8)^{\mathrm{c}}$ \\
\hline 244.6386 & 7420.261 & $5 / 2$ & 48284.498 & $5 / 2$ & 159 & -0.84 & $1.60(8)^{c}$ \\
\hline 245.1477 & 1518.829 & $3 / 2$ & 42298.223 & $3 / 2$ & & -1.04 & $1.02(8)^{\mathrm{c}}, 7.38(7)^{\mathrm{d}}$ \\
\hline 246.6523 & 1518.829 & $3 / 2$ & 42049.478 & $5 / 2$ & 205 & -0.78 & $1.83(8)^{\mathrm{c}}, 1.36(8)^{\mathrm{d}}$ \\
\hline 247.7795 & 6147.085 & $9 / 2$ & 46493.356 & $9 / 2$ & 316 & -0.45 & $3.90(8)^{\mathrm{c}}, 2.84(8)^{\mathrm{d}}$ \\
\hline 248.8769 & 7420.261 & $5 / 2$ & 47588.6 & $3 / 2$ & 262 & -0.34 & $4.90(8)^{\mathrm{c}}, 5.06(8)^{\mathrm{d}}$ \\
\hline 248.9231 & 4716.278 & $7 / 2$ & 44877.209 & $7 / 2$ & & -0.19 & $6.91(8)^{c}, 5.56(8)^{d}$ \\
\hline 249.6636 & 4716.278 & $7 / 2$ & 44758.095 & $9 / 2$ & 142 & -1.00 & $1.08(8)^{\mathrm{c}}, 9.62(7)^{\mathrm{d}}$ \\
\hline 249.7479 & 6147.085 & $9 / 2$ & 46175.395 & $7 / 2$ & 159 & -0.76 & $1.87(8)^{\mathrm{c}}$ \\
\hline & & $5 / 2$ & & $5 / 2$ & & -0.52 & \\
\hline 250.0102 & 18000.627 & $7 / 2$ & 57986.939 & $9 / 2$ & 127 & -0.51 & $3.30(8)^{\mathrm{c}}$ \\
\hline 250.6049 & 28187.578 & $13 / 2$ & 68079.006 & $13 / 2$ & 100 & 0.08 & $1.26(9)^{\mathrm{c}}$ \\
\hline 251.0470 & 19070.550 & $9 / 2$ & 58891.742 & $11 / 2$ & 141 & -0.12 & $7.96(8)^{\mathrm{c}}$ \\
\hline 252.2041 & 4716.278 & $7 / 2$ & 44354.784 & $5 / 2$ & 169 & -0.76 & $1.84(8)^{\mathrm{c}}, 1.49(8)^{\mathrm{d}}$ \\
\hline 253.4822 & 20780.358 & $9 / 2$ & 60219.015 & $11 / 2$ & 128 & -0.12 & $7.81(8)^{\mathrm{c}}$ \\
\hline 255.4853 & 0.000 & $1 / 2$ & 39129.460 & $3 / 2$ & 156 & -1.07 & $8.83(7)^{\mathrm{c}}, 7.13(7)^{\mathrm{d}}$ \\
\hline 255.5095 & 3172.473 & $5 / 2$ & 42298.223 & $3 / 2$ & 22 & -0.70 & $2.05(8)^{\mathrm{c}}, 1.62(8)^{\mathrm{d}}$ \\
\hline 256.3156 & 17436.932 & $11 / 2$ & 56439.643 & $13 / 2$ & 212 & -0.05 & $8.96(8)^{\mathrm{c}}$ \\
\hline 257.1444 & 3172.473 & $5 / 2$ & 42049.478 & $5 / 2$ & 364 & -0.49 & $3.30(8)^{\mathrm{c}}, 2.63(8)^{\mathrm{d}}$ \\
\hline 257.2229 & 20534.191 & $11 / 2$ & 59399.339 & $9 / 2$ & 115 & -0.26 & $5.63(8)^{\mathrm{c}}$ \\
\hline 257.9252 & 23955.349 & $11 / 2$ & 62714.675 & $13 / 2$ & 147 & 0.17 & $1.48(9)^{\mathrm{c}}$ \\
\hline 257.9483 & 26929.008 & $11 / 2$ & 65684.866 & $11 / 2$ & 113 & -0.06 & $8.74(8)^{c}$ \\
\hline 257.9531 & 7420.261 & $5 / 2$ & 46175.395 & $7 / 2$ & 13 & -0.76 & $1.78(8)^{\mathrm{c}}$ \\
\hline 258.9160 & 6147.085 & $9 / 2$ & 44758.095 & $9 / 2$ & 285 & -0.59 & $2.59(8)^{\mathrm{c}}, 1.82(8)^{\mathrm{d}}$ \\
\hline 260.3017 & 16553.087 & $9 / 2$ & 54958.573 & $11 / 2$ & 137 & -0.13 & $7.28(8)^{\mathrm{c}}$ \\
\hline 265.3560 & 4716.278 & $7 / 2$ & 42390.287 & $7 / 2$ & 120 & -1.50 & $3.02(7)^{\mathrm{c}}, 3.03(7)^{\mathrm{d}}$ \\
\hline 265.8032 & 1518.829 & $3 / 2$ & 39129.460 & $3 / 2$ & 139 & -0.77 & $1.63(8)^{\mathrm{c}}, 1.35(8)^{\mathrm{d}}$ \\
\hline 266.4336 & 17436.932 & $11 / 2$ & 54958.573 & $11 / 2$ & 170 & -0.09 & $7.66(8)^{\mathrm{c}}$ \\
\hline 269.7710 & 1518.829 & $3 / 2$ & 38576.313 & $1 / 2$ & 178 & -0.87 & $1.27(8)^{\mathrm{c}}, 9.91(7)^{\mathrm{d}}$ \\
\hline 270.2107 & 19442.466 & $13 / 2$ & 56439.643 & $13 / 2$ & 209 & 0.07 & $1.07(9)^{\mathrm{c}}$ \\
\hline 270.3456 & 31100.286 & $11 / 2$ & 68079.006 & $13 / 2$ & 108 & 0.25 & $1.60(9)^{\mathrm{c}}$ \\
\hline 271.8033 & 16589.603 & $7 / 2$ & 53370.011 & $9 / 2$ & 108 & -0.23 & $5.38(8)^{\mathrm{c}}$ \\
\hline 276.4261 & 0.000 & $1 / 2$ & 36165.356 & $1 / 2$ & 182 & -0.89 & $1.14(8)^{\mathrm{c}}, 9.57(7)^{\mathrm{d}}$ \\
\hline 305.1294 & 13411.939 & $7 / 2$ & 46175.395 & $7 / 2$ & 131 & -0.75 & $1.28(8)^{\mathrm{c}}$ \\
\hline 307.7517 & 23955.349 & $11 / 2$ & 56439.643 & $13 / 2$ & 179 & -0.04 & $6.50(8)^{c}$ \\
\hline 314.5755 & 16553.087 & $9 / 2$ & 48332.758 & $11 / 2$ & 228 & -1.12 & $5.17(7)^{c}$ \\
\hline 320.1579 & 8711.274 & $3 / 2$ & 39936.842 & $5 / 2$ & 178 & -1.68 & $1.37(7)^{\mathrm{c}}$ \\
\hline
\end{tabular}


Table 2. (Continued.)

\begin{tabular}{llllllll}
\hline $\begin{array}{l}\lambda^{\mathrm{a}} \\
(\mathrm{nm})\end{array}$ & $\begin{array}{l}E_{\text {low }}^{\mathrm{b}} \\
\left(\mathrm{cm}^{-1}\right)\end{array}$ & $\mathrm{J}$ & $\begin{array}{l}E_{\text {upp. }}^{\mathrm{b}} \\
\left(\mathrm{cm}^{-1}\right)\end{array}$ & $J^{\prime}$ & Int. $^{\mathrm{b}}$ & $\log g f^{\mathrm{c}}$ & \multicolumn{1}{l}{$\begin{array}{l}{ }^{2} \\
\left(\mathrm{~s}^{-1}\right)\end{array}$} \\
\hline 321.5616 & 11301.024 & $5 / 2$ & 42390.287 & $7 / 2$ & 108 & -1.54 & $1.90(7)^{\mathrm{c}}$ \\
334.3101 & 16589.603 & $7 / 2$ & 46493.356 & $9 / 2$ & 102 & -1.03 & $5.52(7)^{\mathrm{c}}, 6.07(7)^{\mathrm{d}}$ \\
340.6833 & 10592.485 & $3 / 2$ & 39936.842 & $5 / 2$ & 116 & -2.12 & $4.42(6)^{\mathrm{c}}$ \\
341.6625 & 8711.274 & $3 / 2$ & 37971.528 & $3 / 2$ & 296 & -1.72 & $1.10(7)^{\mathrm{c}}$ \\
357.2472 & 10592.485 & $3 / 2$ & 38576.313 & $1 / 2$ & 114 & -1.18 & $3.51(7)^{\mathrm{c}}, 3.39(7)^{\mathrm{d}}$ \\
361.3790 & 14634.336 & $3 / 2$ & 42298.223 & $3 / 2$ & 133 & -1.07 & $4.33(7)^{\mathrm{c}}, 4.60(7)^{\mathrm{d}}$ \\
364.1408 & 8711.274 & $3 / 2$ & 36165.356 & $1 / 2$ & 113 & -1.33 & $2.36(7)^{\mathrm{c}}, 1.98(7)^{\mathrm{d}}$ \\
364.5596 & 14967.745 & $5 / 2$ & 42390.287 & $7 / 2$ & 134 & -1.55 & $1.44(7)^{\mathrm{c}}, 1.17(7)^{\mathrm{d}}$ \\
373.6212 & 18000.627 & $7 / 2$ & 44758.095 & $9 / 2$ & 141 & -1.08 & $3.96(7)^{\mathrm{c}}, 3.42(7)^{\mathrm{d}}$ \\
434.8113 & 13173.337 & $1 / 2$ & 36165.356 & $1 / 2$ & 109 & -1.40 & $1.39(7)^{\mathrm{c}}, 1.01(7)^{\mathrm{d}}$ \\
\hline
\end{tabular}

${ }^{a}$ Observed wavelengths taken from Kramida and Shirai (2006). Vacuum wavelengths for

$\lambda<200.0 \mathrm{~nm}$ and air wavelengths above that limit.

${ }^{\mathrm{b}}$ From Kramida and Shirai (2006).

c From Nilsson et al (2008).

${ }^{\mathrm{d}}$ From Kling et al (2000).

$\mathrm{a}(\mathrm{b})$ is written for $\mathrm{a} \cdot 10^{\mathrm{b}}$.

probabilities of Kling et al (2000) considerably extended and complemented by Nilsson et al (2008). From the comparison with the experimental measurements, it is reasonable to consider that the accuracy of the HFR+CPOL results should be better than $15 \%$ for the most intense transitions, larger uncertainties being possible for the weaker lines particularly for those susceptible to cancellation effects in the calculation of the line strengths.

We give in table 2 a list of selected UV W II transitions suitable for plasma diagnostics. The list is limited to the strongest transitions, i.e. the lines such that the laboratory intensities (Int.) $\geqslant 100$ (Kramida and Shirai 2006). The adopted transition probabilities are those from Kling et al (2000) and Nilsson et al (2008). The transitions for which the calculated $A$-values are likely to be affected by cancellation effects (i.e. for which the cancellation factor $\mathrm{CF}$ as defined by Cowan $(1981)<0.050)$ have been excluded from the list. Note that the line at $400.8751 \mathrm{~nm}$ blended with the W I line at $400.8753 \mathrm{~nm}$ is not included in the table because its calculated line strength was found to be affected by strong cancellation effects. However, it is worth mentioning that this W II line, corresponding to the transition between the even level at $30223.744 \mathrm{~cm}^{-1}(J=3 / 2)$ and the odd level at $55162.390 \mathrm{~cm}^{-1}(J=5 / 2)$, has an estimated $g A$-value of $9.7 \times 10^{6} \mathrm{~s}^{-1}$ (Nilsson et al 2008), i.e. more than one order of magnitude smaller than the transition probability obtained by Den Hartog et al (1987) and Kling and Kock (1999) for the W I line at $400.8753 \mathrm{~nm}\left(\mathrm{~g} A=1.5 \times 10^{8} \mathrm{~s}^{-1}\right)$.

We can see, from the last column of table 2, that the transition probabilities of Kling et al (2000) and Nilsson et al (2008) are in good agreement, as expected, for the 26 transitions common to both works.

\subsection{W III lines}

The only term analysis available in W III has been published by Iglesias et al (1989) who classified 2636 lines between 60 and $268 \mathrm{~nm}$ connecting 235 atomic energy levels.
Very few works have been devoted to radiative rate determinations in doubly ionized tungsten. Schultz-Johanning et al (1999) reported lifetime measurements for three levels obtained with the TR-LIF technique and BFs with the Fourier transform spectroscopy for 81 transitions in the 154-334 $\mathrm{nm}$ spectral range. These authors also proposed transition probabilities for 37 transitions.

More recently, an extensive set of oscillator strengths for W III electric dipole transitions were calculated by Palmeri et al (2008) using the HFR+CPOL method. The accuracy of these new results was assessed through comparisons with the TR-LIF measurements performed by the same authors for two levels belonging to the $5 \mathrm{~d}^{3} 6 \mathrm{p}$ configuration and with the few results of Schultz-Johanning et al (1999). A very limited sample of transition probabilities is presented in the paper of Palmeri et al (2008) but the complete set of results is included in the DESIRE database (4822 transitions in the wavelength range 83-1494 $\mathrm{nm}$ ).

In the present work, we have adopted the transition probabilities of Palmeri et al (2008) and of Schultz-Johanning et al (1999). We report in table 3 a list of selected unblended lines (Iglesias et al 1989) of W III covering basically the UV region and suitable for plasma diagnostics. The list is limited to the strongest transitions, free of cancellation effects and characterized by laboratory intensities (Int.) $\geqslant 250$. It is seen, from the last column of table 3, that the two sets of oscillator strengths agree quite well (for the five lines common to the two papers).

\section{New transition probabilities for forbidden lines}

To our knowledge, no radiative rates have been published so far for forbidden lines in neutral, singly ionized and doubly ionized tungsten. In the present work, transition probabilities have been obtained for magnetic dipole (M1) and electric quadrupole (E2) transitions using the HFR+CPOL approach mentioned above. 
Table 3. UV transitions of doubly ionized tungsten (W III) suitable for plasma diagnostics. For the choice of the lines, see the text.

\begin{tabular}{|c|c|c|c|c|c|c|c|}
\hline $\begin{array}{l}\lambda^{\mathrm{a}} \\
(\mathrm{nm})\end{array}$ & $\begin{array}{l}E_{\mathrm{low}}^{\mathrm{b}} \\
\left(\mathrm{cm}^{-1}\right)\end{array}$ & $\mathrm{J}$ & $\begin{array}{l}E_{\text {upp. }}^{\mathrm{b}} \\
\left(\mathrm{cm}^{-1}\right)\end{array}$ & $J^{\prime}$ & Int. $^{b}$ & $\log g f^{\mathrm{c}}$ & $\begin{array}{l}g A \\
\left(\mathrm{~s}^{-1}\right)\end{array}$ \\
\hline 128.3711 & 10968.54 & 1 & 88867.52 & 0 & 300 & -0.62 & $9.76(8)^{\mathrm{c}}$ \\
\hline 128.5664 & 27252.53 & 3 & 105033.31 & 3 & 300 & -0.30 & $2.03(9)^{\mathrm{c}}$ \\
\hline 129.1862 & & 5 & 97039.60 & 6 & & -0.63 & $9.33(8)^{\mathrm{c}}$ \\
\hline 130.1137 & 12427.09 & 2 & 89283.42 & 1 & 300 & -1.16 & $2.72(8)^{c}$ \\
\hline 131.0199 & 15038.04 & 3 & 91362.35 & 2 & 300 & -0.78 & $6.42(8)^{c}$ \\
\hline 136.1993 & 6277.81 & 3 & 79699.75 & 3 & 300 & -0.91 & $4.37(8)^{\mathrm{c}}$ \\
\hline 137.6777 & 10968.54 & 1 & 83601.96 & 1 & 300 & -0.49 & $1.12(9)^{\mathrm{c}}$ \\
\hline 137.9143 & 6277.81 & 3 & 78786.47 & 3 & 300 & -0.94 & $4.05(8)^{\mathrm{c}}$ \\
\hline 138.1104 & 28977.44 & 4 & 101383.26 & 3 & 300 & -0.40 & $1.41(9)^{\mathrm{c}}$ \\
\hline 138.2742 & 17380.40 & 4 & 89700.42 & 4 & 300 & -0.99 & $3.62(8)^{\mathrm{c}}$ \\
\hline 138.4072 & 17380.40 & 4 & 89630.99 & 5 & 300 & -0.57 & $9.42(8)^{\mathrm{c}}$ \\
\hline 139.9008 & 6277.81 & 3 & 77757.00 & 2 & 500 & -0.84 & $4.92(8)^{\mathrm{c}}$ \\
\hline 140.0876 & 14899.80 & 3 & 86283.75 & 3 & 500 & -0.59 & $8.76(8)^{\mathrm{c}}$ \\
\hline 141.1086 & 16723.24 & 5 & 87590.69 & 4 & 300 & -0.20 & $2.12(9)^{\mathrm{c}}$ \\
\hline 141.7358 & 6277.81 & 3 & 76831.39 & 2 & 500 & -1.20 & $2.10(8)^{\mathrm{c}}$ \\
\hline 141.8356 & 18380 & 6 & & 6 & 50 & -0.29 & \\
\hline 142. & & 5 & & 5 & & -0.34 & $52(9)^{c}$ \\
\hline 143.0391 & 13700.95 & 4 & 836 & 3 & 300 & -0.41 & $26(9)^{\mathrm{c}}$ \\
\hline 143.3444 & 6277.81 & 3 & $76 c$ & 2 & 300 & -0.53 & $.53(8)^{\mathrm{c}}$ \\
\hline 143.9239 & 18380.90 & 6 & 87862.02 & 5 & 250 & -0.26 & $1.74(9)^{\mathrm{c}}$ \\
\hline 145.9855 & 16723.24 & 5 & 85222.97 & 5 & 500 & 0.11 & $3.96(9)^{\mathrm{c}}$ \\
\hline 146.1826 & 22955.03 & 2 & 91362.35 & 2 & 250 & -0.32 & $1.50(9)^{\mathrm{c}}$ \\
\hline 146.5630 & 19632.05 & 5 & 87862.02 & 5 & 250 & -0.59 & $8.03(8)^{\mathrm{c}}$ \\
\hline 146.7436 & 16723.24 & 5 & 84869.01 & 4 & 350 & -0.16 & $2.13(9)^{\mathrm{c}}$ \\
\hline 146.7748 & 12427.09 & 2 & 805 & 3 & 25 & -0.74 & $5.66(8)^{\mathrm{c}}$ \\
\hline 146.8132 & 7686.68 & 4 & 758 & 5 & 40 & -0.48 & $1.03(9)^{\mathrm{c}}$ \\
\hline 147.0641 & 20432 . & 4 & & 3 & 25 & -0.01 & $2.99(9)^{\mathrm{c}}$ \\
\hline 147.1355 & 4461.19 & 2 & & 1 & 400 & -0.32 & $1.47(9)^{\mathrm{c}}$ \\
\hline 147.2613 & 25963 & 6 & & 6 & 00 & 0.27 & 5.71( \\
\hline 147 & & 6 & & 5 & & 0.51 & \\
\hline 147.9 & 1838 & 6 & & 6 & & 0.19 & $71(9)^{\mathrm{c}}$ \\
\hline 148.3 & 62 & 3 & & 3 & 35 & -0.48 & $1.01(9)^{\mathrm{c}}$ \\
\hline 148.9018 & 20432.53 & 4 & 875 & 4 & 250 & -0.24 & $1.72(9)^{\mathrm{c}}$ \\
\hline 149.1151 & 13992.14 & 2 & 81054. & 1 & 250 & -0.39 & $1.21(9)^{\mathrm{c}}$ \\
\hline 149.2185 & 19851.87 & 3 & 86867.50 & 2 & 300 & -0.14 & $2.15(9)^{\mathrm{c}}$ \\
\hline 149.6061 & 18380.90 & 6 & 5222.97 & 5 & 350 & -0.15 & $2.11(9)^{\mathrm{c}}$ \\
\hline 150.1416 & 6277.81 & 3 & 72881.50 & 3 & 450 & -0.55 & $8.38(8)^{\mathrm{c}}$ \\
\hline 150.1897 & 2256.20 & 1 & 8.57 & 0 & 300 & -0.64 & $6.77(8)^{\mathrm{c}}$ \\
\hline 150.3921 & 18376.40 & 4 & 69.01 & 4 & 500 & 0.12 & $3.84(9)^{\mathrm{c}}$ \\
\hline 150.6414 & 23317.80 & 5 & 9700.42 & 4 & 300 & -0.20 & $1.88(9)^{\mathrm{c}}$ \\
\hline 150.8268 & 461.19 & 2 & 70762 . & 2 & 500 & -0.32 & $1.40(9)^{\mathrm{c}}$ \\
\hline 152.0241 & 16723.24 & 5 & & 4 & 450 & -0.04 & $2.67(9)^{\mathrm{c}}$ \\
\hline 152.3764 & & 1 & & 0 & 250 & -0.38 & $1.19(9)^{\mathrm{c}}$ \\
\hline & & 6 & & 5 & & 0.28 & $5.46(9)^{\mathrm{c}}$ \\
\hline & & 4 & & 3 & 30 & -0.09 & \\
\hline 2907 & & 4 & & 3 & 300 & -0.85 & 4.04 \\
\hline 153.3781 & 25963.79 & 6 & 91161. & 5 & 350 & -0.21 & $1.75(9)^{\mathrm{c}}$ \\
\hline 154.1892 & 2256.20 & 1 & 67111.20 & 2 & 250 & -0.72 & $5.32(8)^{c}$ \\
\hline 154.6251 & 16621.08 & 2 & 81293.43 & 2 & 300 & -0.30 & $1.40(9)^{\mathrm{c}}$ \\
\hline 154.9321 & 23317.80 & 5 & 87862.02 & 5 & 250 & -0.13 & $2.06(9)^{\mathrm{c}}$ \\
\hline 155.0009 & 4461.19 & 2 & 68976.80 & 1 & 250 & -0.87 & $3.74(8)^{\mathrm{c}}$ \\
\hline 155.0754 & 6277.81 & 3 & 70762.26 & 2 & 350 & -0.39 & $1.14(9)^{\mathrm{c}}$ \\
\hline 155.3099 & 7686.68 & 4 & 72073.75 & 4 & 500 & -0.06 & $2.42(9)^{\mathrm{c}}$ \\
\hline 155.3608 & 22212.08 & 3 & 86578.41 & 3 & 250 & -0.46 & $9.77(8)^{\mathrm{c}}$ \\
\hline 155.9409 & 13700.95 & 4 & 77827.64 & 4 & 1000 & 0.18 & $4.05(9)^{\mathrm{c}}$ \\
\hline 156.3874 & 6277.81 & 3 & 70221.35 & 4 & 500 & -0.25 & $1.53(9)^{\mathrm{c}}$ \\
\hline 156.8115 & 16723.24 & 5 & 0493.89 & 5 & 300 & -0.30 & $1.36(9)^{\mathrm{c}}$ \\
\hline 157.8008 & 13700.95 & 4 & 77071.73 & 3 & 500 & -0.10 & $2.14(9)^{\mathrm{c}}$ \\
\hline 158.7424 & 7686.68 & 4 & 70681.63 & 3 & 300 & -0.56 & $7.32(8)^{\mathrm{c}}$ \\
\hline 159.0468 & 16723.24 & 5 & 79597.73 & 4 & 400 & -0.26 & $1.46(9)^{\mathrm{c}}$ \\
\hline 159.4222 & & 2 & 67187.3 & 3 & 50 & -0.22 & $1.56(9)^{\mathrm{c}}$ \\
\hline & 23317.80 & 5 & & 6 & 250 & -0.23 & $1.53(9)^{\mathrm{c}}$ \\
\hline 159.6611 & 18380.90 & 6 & 81013.46 & 6 & 500 & -0.08 & $2.18(9)^{\mathrm{c}}$ \\
\hline
\end{tabular}


Table 3. (Continued.)

\begin{tabular}{|c|c|c|c|c|c|c|c|}
\hline $\begin{array}{l}\lambda^{\mathrm{a}} \\
(\mathrm{nm})\end{array}$ & $\begin{array}{l}E_{\mathrm{low}}^{\mathrm{b}} \\
\left(\mathrm{cm}^{-1}\right)\end{array}$ & $\mathrm{J}$ & $\begin{array}{l}E_{\text {upp. }}^{\mathrm{b}} \\
\left(\mathrm{cm}^{-1}\right)\end{array}$ & $J^{\prime}$ & Int. $^{b}$ & $\log g f^{\mathrm{c}}$ & $\begin{array}{l}g A \\
\left(s^{-1}\right)\end{array}$ \\
\hline 159.9109 & 7686.68 & 4 & 70221.35 & 4 & 400 & -0.39 & $1.07(9)^{\mathrm{c}}$ \\
\hline 161.9180 & 17380.40 & 4 & 79139.81 & 3 & 300 & -0.93 & $3.00(8)^{c}$ \\
\hline 162.1131 & 16723.24 & 5 & 78408.35 & 4 & 300 & -0.64 & $5.86(8)^{\mathrm{c}}$ \\
\hline 163.5588 & 14899.80 & 3 & 76039.80 & 2 & 250 & -0.44 & $9.09(8)^{\mathrm{c}}$ \\
\hline 164.3065 & 19632.05 & 5 & 80493.89 & 5 & 250 & -0.38 & $1.04(9)^{\mathrm{c}}$ \\
\hline 165.1104 & 2256.20 & 1 & 62821.85 & 2 & 500 & -0.46 & $8.44(8)^{\mathrm{c}}, 5.25(8)^{\mathrm{d}}$ \\
\hline 166.1248 & 0.00 & 0 & 60195.86 & 1 & 500 & -0.56 & $6.58(8)^{c}, 5.31(8)^{d}$ \\
\hline 166.4965 & 20432.53 & 4 & 80493.89 & 5 & 250 & -0.24 & $1.38(9)^{\mathrm{c}}$ \\
\hline 166.5411 & 7686.68 & 4 & 67731.94 & 5 & 450 & -0.47 & $8.14(8)^{\mathrm{c}}$ \\
\hline 166.5782 & 18376.40 & 4 & 78408.35 & 4 & 250 & -0.38 & $1.01(9)^{\mathrm{c}}$ \\
\hline 166.9669 & 14899.80 & 3 & 74791.95 & 2 & 300 & -0.36 & $1.04(9)^{\mathrm{c}}$ \\
\hline 167.1895 & 13992.14 & 2 & 73804.59 & 1 & 250 & -0.42 & $8.95(8)^{\mathrm{c}}$ \\
\hline 167.2420 & 10968.54 & 1 & 70762.26 & 2 & 250 & -0.50 & $7.67(8)^{\mathrm{c}}$ \\
\hline 169.1430 & 14899.80 & 3 & 74021.35 & 4 & 450 & -0.05 & $2.10(9)^{\mathrm{c}}$ \\
\hline 169.7994 & 23317.80 & 5 & 82210.83 & 5 & 250 & -0.26 & $1.26(9)^{\mathrm{c}}$ \\
\hline 169.8099 & 13992.14 & 2 & 72881.50 & 3 & 350 & -0.28 & $1.22(9)^{\mathrm{c}}$ \\
\hline 170.7752 & 19851.87 & 3 & 78408.35 & 4 & 300 & -0.06 & $1.98(9)^{\mathrm{c}}$ \\
\hline 171.1747 & 17380.40 & 4 & 75800.20 & 5 & 400 & -0.15 & $1.61(9)^{\mathrm{c}}$ \\
\hline 171.4230 & 12427.09 & 2 & 70762.26 & 2 & 400 & -0.21 & $1.42(9)^{\mathrm{c}}$ \\
\hline 171.6602 & 12427.09 & 2 & 70681.63 & 3 & 500 & -0.20 & $1.43(9)^{\mathrm{c}}$ \\
\hline 172.3890 & 10968.54 & 1 & 68976.80 & 1 & 400 & -0.26 & $1.24(9)^{\mathrm{c}}$ \\
\hline 172.8799 & 15038.04 & 3 & 72881.50 & 3 & 450 & -0.32 & $1.06(9)^{\mathrm{c}}$ \\
\hline 172.9329 & 27252.53 & 3 & 85078.28 & 4 & 350 & 0.08 & $2.71(9)^{\mathrm{c}}$ \\
\hline 173.3229 & 23317.80 & 5 & 81013.46 & 6 & 300 & -0.28 & $1.18(9)^{\mathrm{c}}$ \\
\hline 177.5615 & 27252.53 & 3 & 83571.04 & 4 & 300 & -0.13 & $1.59(9)^{\mathrm{c}}$ \\
\hline 178.0368 & 19632.05 & 5 & 75800.20 & 5 & 300 & -0.23 & $1.24(9)^{\mathrm{c}}$ \\
\hline 178.3636 & 19632.05 & 5 & 75697.21 & 6 & 1000 & 0.60 & $8.34(9)^{\mathrm{c}}$ \\
\hline 178.8610 & 17380.40 & 4 & 73289.66 & 5 & 500 & 0.22 & $3.47(9)^{\mathrm{c}}$ \\
\hline 179.5149 & 23080.82 & 1 & 78786.47 & 2 & 300 & -0.38 & $8.70(8)^{\mathrm{c}}$ \\
\hline 181.2144 & 15038.04 & 3 & 70221.35 & 4 & 500 & -0.33 & $9.35(8)^{c}$ \\
\hline 181.5469 & 35429.01 & 6 & 90511.29 & 7 & 800 & 0.72 & $1.06(10)^{\mathrm{c}}$ \\
\hline 181.8859 & 28977.44 & 4 & 83957.03 & 5 & 300 & -0.04 & $1.86(9)^{\mathrm{c}}$ \\
\hline 182.1049 & 18376.40 & 4 & 73289.66 & 5 & 50 & -0.15 & $1.43(9)^{\mathrm{c}}$ \\
\hline 182.6357 & 31211.76 & 5 & 85965.60 & 6 & 300 & 0.36 & $4.56(9)^{\mathrm{c}}$ \\
\hline 190.6880 & 19632.05 & 5 & 72073.75 & 4 & 300 & -0.25 & $1.02(9)^{\mathrm{c}}$ \\
\hline 197.6701 & 19632.05 & 5 & 70221.35 & 4 & 800 & -0.01 & $1.67(9)^{\mathrm{c}}$ \\
\hline 198.6033 & 17380.40 & 4 & 67731.94 & 5 & 1000 & -0.12 & $1.29(9)^{\mathrm{c}}, 9.28(8)^{\mathrm{d}}$ \\
\hline 199.1777 & 14899.80 & 3 & 65106.05 & 4 & 800 & 0.03 & $1.79(9)^{\mathrm{c}}$ \\
\hline 199.7286 & 15038.04 & 3 & 65106.05 & 4 & 800 & -0.96 & $1.82(8)^{\mathrm{c}}$ \\
\hline 200.5799 & 41322.55 & 5 & 91161.95 & 5 & 300 & -0.10 & $1.32(9)^{\mathrm{c}}$ \\
\hline 200.7104 & 17380.40 & 4 & 67187.37 & 3 & 800 & -0.18 & $1.09(9)^{\mathrm{c}}$ \\
\hline 200.7313 & 31211.76 & 5 & 81013.46 & 6 & 500 & -0.18 & $1.09(9)^{\mathrm{c}}$ \\
\hline 204.8067 & 18376.40 & 4 & 67187.37 & 3 & 500 & -0.45 & $5.59(8)^{\mathrm{c}}$ \\
\hline 205.7830 & 33631.43 & 4 & 82210.83 & 5 & 300 & -0.21 & $9.62(8)^{c}$ \\
\hline 207.8347 & 19632.05 & 5 & 67731.94 & 5 & 800 & 0.09 & $1.89(9)^{\mathrm{c}}, 1.64(9)^{\mathrm{d}}$ \\
\hline 209.2755 & 12427.09 & 2 & 60195.86 & 1 & 800 & -0.45 & $5.34(8)^{\mathrm{c}}, 6.15(8)^{\mathrm{d}}$ \\
\hline 214.4516 & 31211.76 & 5 & 77827.64 & 4 & 300 & -0.25 & $7.97(8)^{\mathrm{c}}$ \\
\hline 214.5775 & 14899.80 & 3 & 61488.36 & 3 & 800 & -0.32 & $6.93(8)^{\mathrm{c}}$ \\
\hline 214.586 & 31821.76 & 3 & 78408.35 & 4 & 500 & -0.32 & $6.98(8)^{\mathrm{c}}$ \\
\hline 215.2161 & 15038.04 & 3 & 61488.36 & 3 & 800 & -0.76 & $2.53(8)^{\mathrm{c}}$ \\
\hline 216.0897 & 10968.54 & 1 & 57231.04 & 2 & 500 & -0.10 & $1.15(9)^{\mathrm{c}}$ \\
\hline 218.5424 & 36467.29 & 4 & 82210.83 & 5 & 500 & -0.16 & $9.76(8)^{\mathrm{c}}$ \\
\hline 219.3043 & 35429.01 & 6 & 81013.46 & 6 & 900 & 0.20 & $2.19(9)^{\mathrm{c}}$ \\
\hline 223.1251 & 12427.09 & 2 & 57231.04 & 2 & 500 & -0.47 & $4.56(8)^{\mathrm{c}}$ \\
\hline 225.6009 & 28977.44 & 4 & 73289.66 & 5 & 500 & -0.40 & $5.29(8)^{\mathrm{c}}$ \\
\hline 236.8974 & 31821.76 & 3 & 74021.35 & 4 & 300 & -0.46 & $4.10(8)^{c}$ \\
\hline 257.9570 & 28977.44 & 4 & 67731.94 & 5 & 800 & -0.55 & $2.86(8)^{\mathrm{c}}$ \\
\hline
\end{tabular}

${ }^{a}$ Observed wavelengths taken from Iglesias et al (1989). Vacuum wavelengths for $\lambda<200.0 \mathrm{~nm}$ and air wavelengths above that limit.

b From Iglesias et al (1989).

c From Palmeri et al (2008).

${ }^{\mathrm{d}}$ From Schultz-Johanning et al (2001). $\mathrm{a}(\mathrm{b})$ is written for $\mathrm{a} \cdot 10^{\mathrm{b}}$. 
Table 4. Transition probabilities for forbidden lines in W I. Only transitions for which $A$-values are greater than $0.2 \mathrm{~s}^{-1}$ and $\lambda$ are shorter than $2000 \mathrm{~nm}$ are given.

\begin{tabular}{|c|c|c|c|c|c|c|}
\hline \multirow[b]{2}{*}{$\lambda(\mathrm{nm})^{\mathrm{a}}$} & \multicolumn{2}{|c|}{ Lower level } & \multicolumn{2}{|c|}{ Upper level } & \multirow[b]{2}{*}{ Type } & \multirow[b]{2}{*}{$A_{k i}\left(\mathrm{~s}^{-1}\right)$} \\
\hline & $\mathrm{E}\left(\mathrm{cm}^{-1}\right)^{\mathrm{b}}$ & $\mathrm{J}$ & $\mathrm{E}\left(\mathrm{cm}^{-1}\right)^{\mathrm{b}}$ & $\mathrm{J}$ & & \\
\hline 546.880 & 0.00 & 0 & 18280 & 2 & E2 & $15(+0)$ \\
\hline 552.857 & 0.00 & 0 & 8082.83 & 1 & M1 & $88(-1)$ \\
\hline 568.565 & 1670.29 & 1 & 19253.56 & 2 & E2 & $8.97(-1)$ \\
\hline 577.733 & 1670.29 & 1 & 18974.51 & 3 & E2 & $1.41(+0)$ \\
\hline 601.873 & 1670.29 & 1 & 18280.48 & 2 & $\mathrm{M} 1+\mathrm{E} 2$ & $2.97(-1)$ \\
\hline 607.862 & 1670.29 & 1 & 18116.84 & 2 & $\mathrm{M} 1+\mathrm{E} 2$ & $2.21(+0)$ \\
\hline 609.122 & 1670.29 & 1 & 18082.83 & 1 & $\mathrm{M} 1+\mathrm{I}$ & $6.23(-1)$ \\
\hline 613.242 & 2951.29 & 3 & 19253.56 & 2 & M1 & $1.84(+0)$ \\
\hline 62 & 2951.29 & 3 & 18974.51 & 3 & $\mathrm{M} 1+\mathrm{H}$ & $5.53(-1)$ \\
\hline 62 & 3325.53 & 2 & 19256 & 4 & E2 & 1.11( \\
\hline 6 & & 2 & & 3 & M & \\
\hline & 2951.29 & 3 & 82 & 2 & & \\
\hline & & 0 & 49 & 2 & & \\
\hline & 3325 & 2 & 82 & & & \\
\hline & 332 & 2 & 81 & 2 & & \\
\hline 677.44 & 3325.53 & 2 & 808 & 1 & I1 & \\
\hline 693.119 & 4830.00 & 3 & 19253.56 & 2 & $\mathrm{M} 1+\mathrm{E} 2$ & $2.11(+0)$ \\
\hline 695.429 & 3325.53 & 2 & 17701.18 & 3 & M1- & $9.48(-1)$ \\
\hline 706.793 & 4830.00 & 3 & 8974.51 & 3 & $\mathrm{M} 1+\mathrm{I}$ & $2.11(-1)$ \\
\hline 743.263 & 4830.00 & 3 & 18280.48 & 2 & M1 & $3.67(-1)$ \\
\hline 751.340 & 1670.29 & 1 & 1497 & 2 & $\mathrm{M} 1+\mathrm{I}$ & $2.98(-1)$ \\
\hline 752.417 & 4830.00 & 3 & 18116 & 2 & $\mathrm{M} 1+\mathrm{H}$ & $6.04(-1)$ \\
\hline 776.716 & 00 & 3 & 177 & 3 & M1 & $1.53(+0)$ \\
\hline & & 4 & & 3 & & $3.26(-1)$ \\
\hline & & 3 & & 3 & & \\
\hline & & 3 & & 4 & 1 & $8.98(-$ \\
\hline & & 1 & 1 & 2 & 1 & 1.60( \\
\hline 85 & 33 & 2 & 497 & 2 & $\mathrm{M} 1+\mathrm{I}$ & $6.14(-$ \\
\hline 918.217 & 6219.33 & 4 & 17107 & 4 & M1 & $1.55(+0)$ \\
\hline 956.476 & 3325.53 & 2 & 13777.71 & 2 & M1 & $1.15(-$ \\
\hline 997.429 & 3325.53 & 2 & 13348.56 & 3 & M1 & $8.95(-1)$ \\
\hline 1001.572 & 3325.53 & 2 & 13307.10 & 1 & M1 & $5.36(+0)$ \\
\hline 1081.875 & 6219.33 & 4 & 15460.01 & 3 & M1 & $9.81(-1)$ \\
\hline 1129.558 & 6219.33 & 4 & 15069.93 & 5 & $\mathrm{M} 1+\mathrm{E}$ & $2.09(-1)$ \\
\hline 1173.586 & 4830.00 & 3 & 13348.56 & 3 & M1 & $1.47(+0)$ \\
\hline 1168.619 & 9528.06 & 0 & 18082.83 & 1 & M1 & $2.30(+0)$ \\
\hline 1272.278 & 1670.29 & 1 & 9528.06 & 0 & M1 & $8.89(+0)$ \\
\hline 1363.519 & 4830.00 & 3 & 12161.96 & 4 & M1 & $3.56(-1)$ \\
\hline 1681.214 & 13307.10 & 1 & 19253.56 & 2 & M1 & $2.37(-1)$ \\
\hline 1682.297 & 6219.33 & 4 & 12161.96 & 4 & M1 & $1.27(+0)$ \\
\hline
\end{tabular}

a Air wavelengths deduced from the experimental levels.

${ }^{\mathrm{b}}$ From Kramida and Shirai (2006).

$\mathrm{a}(\mathrm{b})$ is written for $\mathrm{a} \cdot 10^{\mathrm{b}}$.

For W I, the most important forbidden transitions connect the lowest states belonging to the $5 \mathrm{~d}^{4} 6 \mathrm{~s}^{2}$ and $5 \mathrm{~d}^{5} 6 \mathrm{~s}$ evenparity configurations. In our calculations, intra-valence-type interactions were considered by including, in the configuration interaction expansions, the configurations $5 \mathrm{~d}^{4} 6 \mathrm{~s}^{2}, 5 \mathrm{~d}^{5} 6 \mathrm{~s}$, $5 \mathrm{~d}^{5} 7 \mathrm{~s}, \quad 5 \mathrm{~d}^{6}, \quad 5 \mathrm{~d}^{4} 6 \mathrm{~s} 7 \mathrm{~s}, \quad 5 \mathrm{~d}^{4} 6 \mathrm{~s} 6 \mathrm{~d}, \quad 5 \mathrm{~d}^{5} 6 \mathrm{~d}, \quad 5 \mathrm{~d}^{4} 6 \mathrm{p}^{2}, \quad 5 \mathrm{~d}^{4} 6 \mathrm{~d}^{2}$, $5 d^{3} 6 s 6 p^{2}$ and $5 d^{2} 6 s^{2} 6 p^{2}$. Core-valence interactions were taken into account using a core-polarization potential with $\alpha_{d}=4.59 a_{0}^{3}$ as the dipole polarizability of the $\mathrm{W}^{4+}$ ionic core (Fraga et al 1976) and $r_{c}=1.99 a_{0}$ as the cut-off radius, this latter parameter corresponding to the HFR mean value $\langle r\rangle$ of the outermost $5 \mathrm{~d}$ core orbital. The HFR+CPOL method was then combined with a least-squares optimization routine using the energy levels compiled by Kramida and Shirai (2006) for
$5 d^{4} 6 s^{2}$ and $5 d^{5} 6 s$ configurations. The standard deviation of the fit was found to be equal to $73 \mathrm{~cm}^{-1}$.

In W II, the HFR+CPOL approach was also used for calculating the transition rates for forbidden lines involving the energy levels of $5 \mathrm{~d}^{5}, 5 \mathrm{~d}^{4} 6 \mathrm{~s}$ and $5 \mathrm{~d}^{3} 6 \mathrm{~s}^{2}$ even-parity configurations. The same set of interacting configurations as the one considered by Nilsson et al (2008) was included in the model, i.e. $5 d^{5}, 5 d^{4} 6 s, 5 d^{4} 7 s, 5 d^{4} 8 s, 5 d^{3} 6 s^{2}, 5 d^{3} 6 s 7 s$, $5 d^{3} 6 s 8 s, 5 d^{4} 6 d, 5 d^{3} 6 s 6 d, 5 d^{2} 6 s^{2} 6 d$ and $5 d^{3} 6 p^{2}$. The corepolarization potential was chosen by assuming a $4 \mathrm{f}^{14} 5 \mathrm{~d}^{2} \mathrm{Yb}-$ like $\mathrm{W}^{4+}$ ionic core surrounded by three valence electrons. The dipole polarizability tabulated by Fraga et al (1976) for $\mathrm{W} \mathrm{V}$ was adopted, i.e. $\alpha_{d}=4.59 a_{0}^{3}$. The cut-off radius used was the HFR mean radius of the $5 \mathrm{~d}$ orbital in W II, i.e. $r_{c}=$ 
Table 5. Transition probabilities for forbidden lines in W II. Only transitions for which $A$-values are greater than $0.2 \mathrm{~s}^{-1}$ and $\lambda$ are shorter than $2000 \mathrm{~nm}$ are given.

\begin{tabular}{|c|c|c|c|c|c|c|}
\hline \multirow[b]{2}{*}{$\lambda(\mathrm{nm})^{\mathrm{a}}$} & \multicolumn{2}{|c|}{ Lower level } & \multicolumn{2}{|c|}{ Upper level } & \multirow[b]{2}{*}{ Type } & \multirow[b]{2}{*}{$A_{k i}\left(\mathrm{~s}^{-1}\right)$} \\
\hline & $\mathrm{E}\left(\mathrm{cm}^{-1}\right)^{\mathrm{b}}$ & $\mathrm{J}$ & $\mathrm{E}\left(\mathrm{cm}^{-1}\right)^{\mathrm{b}}$ & $\mathrm{J}$ & & \\
\hline 515.214 & 0.000 & $1 / 2$ & 19403.991 & $1 / 2$ & M1 & $1.67(+0)$ \\
\hline & & & & & & \\
\hline 551.76 & 518.829 & $3 / 2$ & & & & \\
\hline 558.96 & 1518.829 & $3 / 2$ & 19403.5 & $1 / 2$ & $\mathrm{M} 1+\mathrm{E}$ & $36(-1)$ \\
\hline 562.98 & 1518.829 & $3 / 2$ & 276. & $5 / 2$ & M1 & \\
\hline 572.182 & 3.829 & $3 / 2$ & 990.9 & $3 / 2$ & M1 & \\
\hline 607.187 & 3172.473 & $5 / 2$ & 19637.309 & $5 / 2$ & M1 & $2.93(-1)$ \\
\hline 620.794 & 3172.473 & $5 / 2$ & 19276.431 & $5 / 2$ & M1 & $2.02(-1)$ \\
\hline 631.998 & 3172.473 & $5 / 2$ & 18990.929 & $3 / 2$ & M1 & $9.32(-1)$ \\
\hline 670.010 & 4716.278 & $7 / 2$ & 19637.309 & $5 / 2$ & M1 & $4.37(-1)$ \\
\hline 686.617 & 4716.278 & $7 / 2$ & 19276.431 & $5 / 2$ & M & $2.05(-1)$ \\
\hline 758.90 & 0.000 & $1 / 2$ & 13173.337 & $1 / 2$ & M1 & $3.92(-1)$ \\
\hline & 1518.829 & $3 / 2$ & 13173.337 & $1 / 2$ & M1 & $7.65(-1)$ \\
\hline & 8711.274 & $3 / 2$ & & $5 / 2$ & M1 & $9.12(-1)$ \\
\hline 934 & 711.274 & $3 / 2$ & & $1 / 2$ & $\mathrm{M} 1+\mathrm{E}$ & $5.35(-1)$ \\
\hline & 711.274 & $3 / 2$ & & $3 / 2$ & M1 & \\
\hline & 73 & $5 / 2$ & & $7 / 2$ & & -1) \\
\hline & 78 & $7 / 2$ & & $9 / 2$ & & \\
\hline & 518.829 & $3 / 2$ & & $5 / 2$ & M1 & -1) \\
\hline & 0.000 & $1 / 2$ & 28 & $1 / 2$ & M1 & +0) \\
\hline 4.569 & 10592.485 & $3 / 2$ & 19403.991 & $1 / 2$ & M1 & -0) \\
\hline 1146.765 & 4716.278 & $7 / 2$ & 3434.070 & $5 / 2$ & $\mathrm{M} 1+\mathrm{E}$ & $2.62(-1)$ \\
\hline 1147.782 & 147.085 & $9 / 2$ & 7.160 & $9 / 2$ & M1 & $2.23(+0)$ \\
\hline 1149.68 & 716.278 & $7 / 2$ & 411.939 & $7 / 2$ & M & $11(+0)$ \\
\hline 1151.235 & 10592.485 & $3 / 2$ & 19276.431 & $5 / 2$ & M & $2.07(-1)$ \\
\hline 1190.371 & 10592.485 & $3 / 2$ & 18990.929 & $3 / 2$ & M & $1.61(+0)$ \\
\hline 1229.895 & 172.473 & $5 / 2$ & 11301.0 & $5 / 2$ & M1 & $3.05(-1)$ \\
\hline 1300.051 & 11301.024 & $5 / 2$ & 18990.929 & $3 / 2$ & M & \\
\hline 1347.338 & 172.473 & $5 / 2$ & 10592.4 & $3 / 2$ & M1 & $1.19(+0)$ \\
\hline 1366.886 & 1518.829 & $3 / 2$ & 8832.728 & $1 / 2$ & M1 & $2.83(+0)$ \\
\hline 1604.530 & 13173.337 & $1 / 2$ & & $1 / 2$ & M1 & $4.76(-1)$ \\
\hline & & $5 / 2$ & & $5 / 2$ & M1 & $4.92(-1)$ \\
\hline & 7420.261 & $5 / 2$ & & $5 / 2$ & M & $8.92(-1)$ \\
\hline & 13411.939 & $7 / 2$ & & $5 / 2$ & M & $2.12(-1)$ \\
\hline & & $1 / 2$ & & $3 / 2$ & M & $9.93(-1)$ \\
\hline 1998.266 & 14634.336 & $3 / 2$ & 19637.309 & $5 / 2$ & M1 & $3.73(-1)$ \\
\hline
\end{tabular}

${ }^{a}$ Air wavelengths deduced from the experimental levels.

${ }^{\mathrm{b}}$ From Kramida and Shirai (2006).

a(b) is written for $\mathrm{a} \cdot 10^{\mathrm{b}}$.

$1.77 a_{0}$. The HFR+CPOL method was then combined with the least-squares optimization routine using experimental energy levels published in the recent compilation of Kramida and Shirai (2006) for $5 d^{5}, 5 d^{4} 6 s$ and $5 d^{3} 6 s^{2}$ configurations. The standard deviation of the fit was found to be equal to $99 \mathrm{~cm}^{-1}$.

The HFR+CPOL method was also considered for computing the transition probabilities for $\mathrm{M} 1$ and $\mathrm{E} 2$ transitions within the $5 \mathrm{~d}^{4}, 5 \mathrm{~d}^{3} 6 \mathrm{~s}$ and $5 \mathrm{~d}^{2} 6 \mathrm{~s}^{2}$ lowest configurations of W III. In this case, the valence-valence interactions were taken into account by including, in the theoretical model, the same configurations as those included by Palmeri et al (2008), i.e. $5 d^{4}, 5 d^{3} 6 s, 5 d^{3} 7 s, 5 d^{3} 8 s, 5 d^{2} 6 s^{2}$, $5 d^{2} 6 s 7 s, 5 d^{2} 6 s 8 s, 5 d^{3} 6 d, 5 d^{2} 6 s 6 d, 5 d 6 s^{2} 6 d$ and $5 d^{2} 6 p^{2}$. The core-polarization potential used a $4 \mathrm{f}^{14} 5 \mathrm{~d}$ Tm-like $\mathrm{W}^{5+}$ ionic core surrounded by three valence electrons. As no dipole polarizability was tabulated by Fraga et al (1976) for W VI, a value of $4.00 a_{0}^{3}$ was extrapolated along the Tm isoelectronic sequence. The cut-off radius used was the HFR mean radius of the $5 \mathrm{~d}$ orbital in W III, i.e. $1.66 a_{0}$. Then a leastsquares fitting procedure was applied using the energy levels published by Iglesias et al (1989) for the $5 d^{4}, 5 d^{3} 6 s$ and $5 d^{2} 6 s^{2}$ configurations. The standard deviation of the fit was found to be equal to $122 \mathrm{~cm}^{-1}$.

The computed transition probabilities, $A_{k i}$, for M1 and E2 lines connecting the metastable levels in W I, W II and W III are listed in tables 4, 5 and 6, respectively. If the two types of radiation contribute significantly to the total intensity of a line, the sum of both components is given. The exclusion criterion of one particular type of radiation for a given transition is that the corresponding $A$-value should be less than $1 \%$ of the sum of M1 and E2 contributions. Owing to the extensive nature of the results, only transitions for which $A_{k i}$ is greater than $0.2 \mathrm{~s}^{-1}$ and $\lambda$ shorter than $2000 \mathrm{~nm}$ are reported in the tables. 
Table 6. Transition probabilities for forbidden lines in W III. Only transitions for which $A$-values are greater than $0.2 \mathrm{~s}^{-1}$ and $\lambda$ are shorter than $2000 \mathrm{~nm}$ are given.

\begin{tabular}{rrrrrlll}
\hline & \multicolumn{2}{c}{ Lower level } & \multicolumn{2}{c}{ Upper level } & & \\
\cline { 2 - 4 }$\lambda(\mathrm{nm})^{\mathrm{a}}$ & $\mathrm{E}\left(\mathrm{cm}^{-1}\right)^{\mathrm{b}}$ & $\mathrm{J}$ & & $\mathrm{E}\left(\mathrm{cm}^{-1}\right)^{\mathrm{b}}$ & $\mathrm{J}$ & Type & $A_{k i}\left(\mathrm{~s}^{-1}\right)$ \\
\hline 695.950 & 2256.20 & 1 & 16621.08 & 2 & $\mathrm{M} 1+\mathrm{E} 2$ & $5.44(-1)$ \\
736.496 & 6277.81 & 3 & 19851.87 & 3 & M1+E2 & $4.29(-1)$ \\
821.792 & 7686.68 & 4 & 19851.87 & 3 & M1+E2 & $6.47(-1)$ \\
822.150 & 4461.19 & 2 & 16621.08 & 2 & M1+E2 & $2.06(-1)$ \\
826.315 & 6277.81 & 3 & 18376.40 & 4 & M1 & $7.69(-1)$ \\
851.849 & 2256.20 & 1 & 13992.14 & 2 & M1 & $1.76(+0)$ \\
935.222 & 7686.68 & 4 & 18376.40 & 4 & M1+E2 & $1.82(+0)$ \\
945.202 & 4461.19 & 2 & 15038.04 & 3 & M1 & $8.16(-1)$ \\
957.719 & 4461.19 & 2 & 14899.80 & 3 & M1+E2 & $5.40(-1)$ \\
966.547 & 6277.81 & 3 & 16621.08 & 2 & M1 & $2.00(+0)$ \\
1048.926 & 4461.19 & 2 & 13992.14 & 2 & M1 & $1.48(+0)$ \\
1106.313 & 7686.68 & 4 & 16723.24 & 5 & M1 & $3.22(-1)$ \\
1141.210 & 6277.81 & 3 & 15038.04 & 3 & M1 & $1.60(+0)$ \\
1159.508 & 6277.81 & 3 & 14899.80 & 3 & M1+E2 & $8.27(-1)$ \\
1187.346 & 4461.19 & 2 & 12881.03 & 1 & M1 & $4.73(+0)$ \\
1307.157 & 2256.20 & 1 & 9904.30 & 0 & M1 & $9.62(+0)$ \\
1346.771 & 6277.81 & 3 & 13700.95 & 4 & M1 & $4.76(-1)$ \\
1536.304 & 4461.19 & 2 & 10968.54 & 1 & M1+E2 & $2.16(-1)$ \\
1662.258 & 7686.68 & 4 & 13700.95 & 4 & M1 & $1.68(+0)$ \\
1706.097 & 13992.14 & 2 & 19851.87 & 3 & M1 & $1.91(+0)$ \\
\hline
\end{tabular}

a Air wavelengths deduced from the experimental levels.

${ }^{b}$ From Iglesias et al (1989).

$\mathrm{a}(\mathrm{b})$ is written for $\mathrm{a} \cdot 10^{\mathrm{b}}$.

\section{Conclusion}

Critically evaluated transition rates available in the literature for allowed electric dipole transitions together with a new set of computed $A$-values for forbidden lines are reported in the present work for W I, W II and W III. These results are intended to provide plasma physicists with some of the data they need for spectroscopic diagnostics and modelling of fusion plasmas magnetically confined in reactors where tungsten is expected to be used as a facing material.

\section{Acknowledgments}

PQ, PP and EB are, respectively, Senior Research Associate, Research Associate and Research Director of the FRSFNRS. Financial support from the ADAS-EU project is acknowledged. VV was financially supported by a grant from the Université Claude Bernard (Lyon, France) and is grateful to colleagues from the Mons group for their kind hospitality.

\section{References}

Cabeza M I, Iglesias L and Rico F R 1985 Opt. Pura Apl. 181 Campbell-Miller M D and Simard B 1996 J. Opt. Soc. Am. B 132115

Clawson J E and Miller M H 1973 J. Opt. Soc. Am. 631598

Corliss C H 1969 J. Res. Natl Bur. Stand. A 73277

Corliss C H and Bozman W R 1962 Natl Bur. Stand. Monograph vol 53 (Washington DC: US Govt Printing Office)

Cowan R D 1981 The Theory of Atomic Structure and Spectra (Berkeley, CA: University of California Press)
Den Hartog E A, Duquette D W and Lawler J E 1987 J. Opt. Soc. Am. B 448

Duquette D W, Salih S and Lawler J E 1981 Phys. Rev. A 242847

Ekberg J O, Kling R and Mende W 2000 Phys. Scr. 61146

Federici G et al 2001 Nucl. Fusion 411967

Fivet V, Quinet P, Palmeri P, Biémont E and Xu H L 2007 J. Electron Spectrosc. Relat. Phenom. 156-8 250

Fraga S, Karwowski J and Saxena K M S 1976 Handbook of Atomic Data (Amsterdam: Elsevier)

Henderson M, Irving R E, Matulioniene R, Curtis L J, Ellis D G, Wahlgren G M and Brage T 1999 Astrophys. J. 520805

Iglesias L, Cabeza M I, Rico F R, Garcia-Riquelme O and Kaufman V 1989 J. Res. Natl Inst. Stand. Tech. 94221

Kling R, Ekberg J O and Kock M 2000 J. Quant. Spectrosc. Radiat. Transfer 67227

Kling R and Kock M 1999 J. Quant. Spectrosc. Radiat. Transfer 62129

Kramida A E and Shirai T 2006 J. Phys. Chem. Ref. Data 35423

Kwiatkowski M, Micali G, Werner K, Schmidt M and Zimmermann P 1982 Z. Phys. A 304197

Kwiatkowski M, Naumann F, Werner K and Zimmermann P 1984 Phys. Lett. A 10349

Laun D D and Corliss C H 1968 J. Res. Natl Bur. Stand. A 72609

Lipschultz B et al 2001 J. Nucl. Mater. 290293286

Martin W C, Zalubas R and Hagan L 1978 Natl Bur. Stand. Monograph vol 60 (Washington DC: US Department of Commerce)

Neu R et al 2005 Nucl. Fusion 45209

Nilsson H, Engström L, Lundberg H, Palmeri P, Fivet V, Quinet P and Biémont É 2008 Eur. Phys. J. D 4913

Obbarius H U and Kock M 1982 J. Phys. B: At. Mol. Phys. 15527

Palmeri P, Quinet P, Fivet V, Biémont E, Nilsson H, Engström L and Lundberg H 2008 Phys. Scr. 78015304

Plekhotkin G A and Verolainen Ya F 1985 Opt. Spectrosc. 58447

Pospieszczyk A 2006 Nuclear Fusion Research (Berlin: Springer) 
Quinet P, Palmeri P, Biémont E, McCurdy M M, Rieger G,

Pinnington E H, Wickliffe M E and Lawler J E 1999 Mon. Not. R. Astron. Soc. 307934

Schnabel R and Kock M 1997 Z. Phys. D 4131

Schnabel R, Schultz-Johanning M and Kock M 1998 Eur. Phys. J. D 4267
Schultz-Johanning M, Schnabel R and Kock M 1999 Eur. Phys. J. D 5341

Shadmi Y and Caspi E 1968 J. Res. Natl Bur. Stand. A 72757

Skinner C H 2008 Can. J. Phys. 86285

Skinner C H 2009 Phys. Scr. T134 014022

Wyart J-F 1978 Phys. Scr. 1887 